\title{
Cher das Vorkommen, Verhalten und die Verbreitung von glatter Muskulatur in der Haut des Mensehen.
}

\author{
Von \\ Paul Quast, \\ Assistout am Anatonisehen Institut Bunn. \\ Wit 6 Textabhildungen. \\ Eingegangen om 4. Mai 19:2.
}

Glatte Muskelfasern kommen in der Haut des Menschen weit verbreitet vor and finden sich, auBer in der Tunica media der HautgefäBe und auBer in der Wand der Schweilidüusen (Myoepithelien), nomalerweise in verschiedener Ausdehnung und Form:

1. Als schiefe glatte Hautmuskeln, zu BündeIn gruppiert, an allen behaarten Hautstellen, - , wogegen sie an unbehaarten Teilen der Haut, wie in der Handfläche und der Fußsohle, gänzlich fehlen“"1), - von Eylandt wegen der aufrichtenden Wirkung ihrer Kontraktion auf die Haarbälge als, , Arrectores pilorum ": von Moleschot, Sappey, Hesse als, "Pressores sebi" bezeichnet, wegen der mit ihrer Kontraktion einhergehenden Druckwirkung auf die Talgdrüsen ron Bonnet ${ }^{\bar{p}}$ ) „Haarbalgdrüsenmuskeln" genannt.

2. Mit einem vorzugsweise zur Oberflüche parallelen Verlauf als ausge. dehntere, flächenhaft ausgebreitete und netzförmig angeordnete Muskellager, in zusammenhängenden Schichten, as sogenannte Fleischhäute: beim männ. lichen Geschlecht in ganz besonderer Mäehtigkeit in der Tunica dartos des Scrotum, ausstrahlend in die Haut des Penis und in die vorderen Teile des Perineum; beim weiblichen Geschlecht (aber auch beim männlichen sich findend) vorwiegend in der Mamilla und in der Areola mammae.

Hiermit scheint das Vorkommen der glatten Muskulatur in der Haut zunähst. erschöpft. Einzelne, spärliche, nicht immer klare und eindeutige Hinweise in wenigen anatomischen und histologischen Lehrbüchern und in der Literatur lassen jedoch eine ausgedehntere Verbreitung der glatten Muskulatur in der Haut über die oben gezogenen Grenzen hinaus vermuten. Nach Rauber-Kopsch ${ }^{37}$ ) werden glatte Muskeln der Haut, , die nicht an Haarbälge gebunden sind, zwar von den meisten bestritten, hierbei ist von der Tunica dartos abgesehen":

Von älteren Untersuchungen, die sich mit der Anatomie und Physiologie der Hautmuskeln beschäftigt haben, verdienen zunächst die Arbeiten von Neumann and Tomsa Erwähnung.

Neumann ${ }^{99-32^{2}}$ ) untersuchte die verschiedenen Gegenden der Haut an 60 Kadavern auf organische Muskelfasern*). Die Ergebnisse seiner Untersuchungen sind zusammengefaßt folgende:

*) Als organische Mnskeln wurden früher die Muskelfizern an Eingeweiden, im. Gegensatz zu den animalischen Muskeln, den skelettmuskeh, bezeichnet. 
"AuBer an den bisher bekannten stellen funden sich teils in der Nähe def: Gefäbe und Nerven, teils vereinzelt im Bindegewebe, zumeist netzförmig zusimmenhängend, an den verschiedensten Stellen der Haut noch organische Muskeln:

1. Selbständige Züge, die am oberen Teile des Corium entspringen und bis zum Panniculus adiposus sich erstrecken, während ihres Verlaufes sich vielfach teilen und sowohl horizontale als vertikale Nebenäste aussenden. Es darf nicht bezweifelt werden, daß es sich hier um selbständige Muskelzüge handelt, welche mit den Muskeln des Haarbalges gar nichts gemein haben.

2. Selbständige, horizontal verlaufende Äste und Stämme, sowohl oberhalh als auch unterhalb der Schweißdrüsen, insbesondere in der behaarten Kopthaut und zuweilen auch in der Achselhöhle. Der Befund in der Achselhöhle ist jedoch nicht allgemein gültig, er ist individuell und lokal variabel; in zahlreich angefertigten Präparaten findet er sich nur bei einzelnen derselben. Es ist in vielen Fällen zweifelhaft, ob man es hier mit selbständigen Muskelfasern zu tun hat, vielleicht gehören die Züge dem System der Arrectores pilorum an.

3. Horizontale, breite Züge in dem oberen Teile des Corium, vorzugsweise in der Kopfhaut und an den Streckflächen der Extremitäten; trägt man die Epidermis vorsichtig ab und macht hierauf fejne Horizontalschnitte, so läBt sich der Verlauf derselben dicht unter den Papillen deutlich erkennen."

„,Die Entwicklung der Körperstärke hat nach Veumann keinen EinfluB auf die Entwicklung der glatten Muskelzüge in der Haut; es findet sich oft die stärkste Hautmuskulatur bei den abgemagertsten Körpern. Bezüglich der Hüufigkeit des Vorkommens der Muskelzüge in den verschiedenen Iokalitiiten der Haut stellt Veumann folgende Skala auf: Scrotum, Penis, Perineum, Warzenhof, Brustwarze, Kopfhaut, Vorderarm, Obersehenkel, Oberarm, Schulter, Stirn, Bauchwand, Achselhöhle, Unterschenkel, Gesicht, Volar- und Dorsalfläche der Hände und Füße. An der inneren Handfläche und Fußsohle konnten keine Muskeln nachgewiesen werden. Ferner sind sie an der Streckseite der Extremitäten stärker entwickelt als an der Beugeseite*). An jenen Lokalitäten, an denen das elastische Gewebe stärker entwickelt ist, wie z. B. in der Kopfhaut, Streckfläche der Extremitäten, sind cie Züge der organischen Muskelfasern ebenfalls mächtiger entwickelt, sowohl der längeren Ausdehnung als der Dicke des Durchmessers nach."

Tomsa ${ }^{51}$ und 51 a), der die anatomische Anordnung der glatten Hautmuskeln untersuchte, teilt diese in drei Gruppen ein, ihre Zugrichtung berücksichtigend:

a) ,Die Zugrichiung des Muskels verläuft parallel der Hautoberfläche, seine Lage und Befestigung ist in der Pars reticularis cutis; hierher gehören die flächenhaft ausgebreiteten und die aus einer, aus netzförmig anastomosierenden und verflochtenen Bündeln bestehenden Schicht gebildeten Fleischhäute (Tunica dartos, Muskelhaut des Penis, Warzenhof).

*) Als Ergänzung zu Neumanns Ergebnissen diene eine dieshezïgliche Ammerkung aus: W. Rrause, Handbuch der menschlichen Anatomie. 3. Aufl. 1876. Hannover, S. 113 ,Manche Ausläufer (der Mm. arrectores pilorum) reichen tiefer in das Unterhautbindegewebe, andere erstrecken sich mehr horizontal an den Schweißdrizsen vorbei. Namentlich in der behaarten Kopfhaut, an der Yorderfläche des Obersehenkels, anch in der Achselhöhle kominen solehe Bündel vor; an den Beugeflachen der Extrenitüten sind sie schwächer entwickelt als an den Streckseiten." 


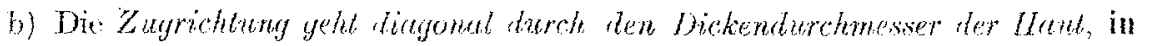
allan möglichon Richinngen, rlie Labe und Befestigung des Muskels ist in rer Par's papillaris und den oberen zwei Dritteilen der Pars reticularis gelegen. Hicher gehören die Hautmuskeh des Gesichts, besonders in der Backen- und Wangengegend." Tomsa nennt sie die Muskelnetze der Haut.

c) "Die Zugrichung ist diagonul aurch die Dicke der Haut in eimer zu: Haarbaigneigung bestimmten Richtung; die Lage und Befestigung ist so, da 3 beide Muskelenden innerhalb der Pars papillaris gelegen sind; zuweilen strahlt das eine Ende pinsel- und fächerförmig in die Hantpapillen aus, das andere greift mit einem umschriebenen Ansatze ara Haar-oder Drüsenbalge, einer Einstillpung des Papillarteiles, an." Die hierher gehörigen Muskeln nennt Tomsa die ,Hebelmuskeln" der Cutis. ,Manchmal werden auch Kombinationen der Muskelnetze mit den Hebelmuskeln wahrgenommen."

Die der Arbeit Tomsas beigefügten Abbildungen zeigen, siob kreuzende und in den versehiedensten Ebenen und Tiefen anastomosierende Muskelbündel und Muskelnetze" und sind zum Teil aus der Backengegend der Gesichtshaut des Yenschen genommen. Tm eine Entfernung des kollagenen Gerüstes der Haut zu erzielen, wurden die Hautstücke in einem Gemiseh von Alkohol und 0,8 bis 1 \% Salzsiure gekocht, nachher angewissert und mit Carmin leicht gefirbt. DaB as sich aber hierbei, in allen Fillen, ausschlieBlich um glatte Muskulatur handele, wird weder durch ten Text der Abhandlung, noch turch die isolicrende urd färberische Methode, noch durch die Abbildungen selbst bewiesen oder gar nur wahrscheinlich gemacht. Besonders die Angaben über die färberische und präparatorische Sicherstellung der muskuliiren Beschaffenheit der fraglichen Faserzüge sind recht unbefriedigend. Vorkommen und Verbreitung der glatten Hautmuskeln in der von Tomsa beschriebenen und bildich wiedergegebenen Form sind zu ausgedehnt, als daß sie dem Histologen und Dermatologen je hätten entgehen können. In einzelnen Fällen scheint es sich um eine Ferkennung und Verwechslung der fraglichen Stränge, Züge und Netze mit dem bindegewebigen und elastischen Gerüstwerke der Cutis zu handeln, während in anderen Fillen Stellungnahme und Entzcheidung schwer und die Möglichkeit, daß Tomsa glatte Muskelzüge gesehen, zugestanden werłen muls.

In seiner Schrift über die Hautkrankheiten erwähnt Jarischir), daB, ,nach Tomsa und auch nach Tnna in vielen Gegenden der Haut (Stirn. Wangen, Rücken) schräg in die Cutis aufsteigende glatte Muskeln reichlich rorkommen, welche aber mit Haarbälgen nicht in Verbindung stehen".

Und nach Klemensiewio ${ }^{19}$ ), kommen an vielen Stellen der Haut, besonders an solchen mit nicht scharfer Spaltbarkeit glatte Muskeln vor, welche eine netzförmige Anordnung zeigen", "Viele Haare", sagt Klemensiearicz, ,besitzen gar keine Arrectores, so z. B. die Cilien, Augenbrauen, Vibrissen, die Härchen der Augenlider, der Nase, der Lippen und die Haare solcher Gegenden, welche reich sind an anderen, glatten und quergestreiften Muskelfasern (Backen, Lippenund Stirngegend*). Andererseits kommen an Stellen, die nur mit Flaumbaaren

*) Xach den Untersuchungen von Diesing $y^{3}$ ) feblt ein Arrector auch den groBen, stets alleinstehenden Haaren am Kinn, obwohl dieselben nit Talydrüsen versehen sind. Möglicherweise übernebmen hier die bis in die oberen Schichten des Corim einstrahlezden, quergestreiften Muskelfasern die Funktionen des Arredtor." 
heselat sind and eine unhestimmte Spalbarkeit besitzen, schräg anfsteigende Muskeln vor, welche aber in kciner direkten Beziehung zu den Haaren stehen."

Unnd $\left.{ }^{52}\right)$ erscheint es am zweckmäBigsten, die glatten Muskeln der Haut in 3 Gruppen zu teilen, in:

1. Membranae seu tunicae musculares;

2. Musculi erectores pilorum;

3. Mrusuli cutis diagonales.

Ad 3 bemerkt Unna: ,Viele Bezirke der Haut (Stirn, Wangen, Rücken) und zwar besonders solche, welche sich durch das Vorhandenscin von Furchen auszeichnen, die in mannigfaltigen und unbestimmten Richtungen verlaufen, sind reich an glatten Muskeln, welche schräg in der Cutis aufsteigen; diese haben mit Rücksicht auf den Zug, welchen sie auf die Haut ausiiben, ganz dieselbe Bedeutung wie die Mm. erectores, wenn sie auch nicht in direkte Beziehungen zu den diesen Hautgegenden eigentümlichen, zarten und dünnen Haaren, Wollhärchen, treten." Unna macht den Vorschlag, sowohl die Mm. erectores, als auch die freien, schräg und diagonal verlaufenden Muskeln der Haut, die Mm. cutis diagonales, als schräge Hautspanner, $M m$. tensores cutis obliqui, zu bezeichnen; (also sowohl diejenigen, welche an den Haarfollikeln einen fixen Anhaltspunkt besitzen, Mrm. erotores pilorum, als auch diejenigen, welche desselben entbehren, Mm. diagonales; - in Gegensatz hierzu setzt Unno dann die Fleischhäute, die Membranen bilkenden Muskeln, die horizontalen Spannmuskeln, Membranae seu tunicae musculares.)

Hierzu sagt Koelliker ${ }^{20}$ ): ,Glatte Muskeln, die nicht an Haarbälge gehen, welche Unna reichlich in der Haut der Stirn, der Wange und des Rückens beschreibt, habe ich noch nicht gesehen; Lnna hat möglicherweise Muskeln, die an große Talgdrüsen gehen, die kleine Härchen enthalten, wie ich solche an den Wangen sah, für selbständige Muskelbündel genommen."

Zur Frage des Vorkommens der glatten Muskulatur in der Haut nimmt Babes ${ }^{2}$ ) wie folgt Stellung: „Die Haut beherbergt drei funktionell verschiedene, ausgebreitete Muskellager, deren ausgebreitetstes wohl jenes ist, welches einen Bestandteil der Gefäßwand bildet. Weiterhin bilden die Arrectores pilorum glatte Muskelfasern und endlich ist ein dritter muskulöser Apparat in cler Tiefe der Haut, besonders der Genitalsphäre (Scrotum, Penis, große Schamlippen, Brustdrüse), dann im Gesicht als ein dünnes Netzwerk von Muskelbalken gelegen. Ahnliche, unbedeutende Züge dieses Gewebes fand ich (Babes) inkonstant in der Umgebung des Afters, des Nabels und des Ohres." *)

In der neueren Literatur finden sich eindeutige und klare Angaben mit Hinweisen auf das Vorkommen von glatter Muskulatur in der Haut des Menschen nur in dem Merkelschen Lehrbuch der Anatomie des Menschen²): „Glatte Muskelzellen", so sagt Merkel, ,stehen meist zu den Haarbägen in Beziehung. Doch findet Häggqvist inmitten des Corium auch horizontal verlaufende Bündel, welche mit solchen nicht in Verbindung stehen."

Gelegentlich histophysiologischer Studien über die Temperatursinne machte Häggqvist ${ }^{11}$ ) systematische Untersuchungen der Haut des Menschen. ,Schon

*) Nach $R o u x^{41}$ ) sind die ,Muskelfasem der Analregion zum Teil Abkömmlinge der Muscularis mucosae und äußeren Längsfasersehicht des Mastdarmes". 
in meinem exsten Priparat", schribt Hägyovis, ,weckte meine Aufmerhamkeit, als ich einen ausgenommenen Kältepunkt untersuchte, ein ganz dickes Bündel glatter Maskelfasern, das ich etwa in derselben Tiefe wie das Rete cutaneum der Hautgefäße liegen sah. Als ich diesen Muskel näher untersuchte, fand ich, daß er weder mit einem Haarfollikel noch mit dem Corpus papillare in etwaige Verbindung trat, sondern daß er immer auf derselben Höhe liegen blieb. Dies machte es ja ganz unwahrscheinlich, daß ich einen $\mathrm{M}$. arrector pili vor mir hätte;" ... ich muß vielmehr glauben, , daß es sich in diesem Falle um einen vorher nicht bekannten Muskel handelte".

Uber das Vorkommen dieses Muskels berichtet Häygqvist weiter: ,Später habe ich immer, ohne jegliche Ausnahme (s. doch weiter unten über Vola manus und Planta pedis), ein Bündel glatter Muskulatur gefunden, wenn ich einen Kältepunkt untersucht habe. Wenn ich aber andere Hautstüche ausgeschnitten habe (was vielfach geschehen ist), konnte ich andererseits nie einen solchen Muskel wiederfinden. Das Aussehen des Wuskels variiert ein wenig in verschiedenen Präparaten."

Häggqvist beschreibt nun diese Muskeln, ,im Anschluß an einige Abbildungen ausgewïhlter Sohnitte, in Serien geordnet": ,Abb. $\stackrel{\Xi}{*}$ und folgende zeigen ein glattes Muskelbundel, das gegen die Hautoberfliche eine perpendikuläre Richtung einnimmt, also dieselbe Richtung, wie man von einem ML arrector zu erwarten hätte. Doch reicht es nicht bis zum Corpus papillare auf und ist auch nicht an einen Haarbalg gebunden. In den folgenden Schnitten nimmt das Bündel ab. um später sowohl an Länge wie an Dicke wieder zuzunehmen. Gleichzeitig nimmt das Bündel nun eine neue Richtung ein, indem es mehr sehief gegen die Oberfläche läuft. In den folgenden Schnitten nimmt das Bündel wieder ab, bis man endlich nur einen kleinen Knoten sieht. Von diesem Knoten entwickelt sich dann ein Bündel, das eine Iage parallel zu der des vorigen Bündels einnimmt. Dies Bündel, das bald abnimmt und versehwindet, ist etwa von derselben Dicke wie das zuerst beschriebene, zur Haut perpendikuläre Bündel, das sich über 5 ron den $10 \mu$ dicken Schnitten streckt, das zweite geht über 17 und das letzte über 12 Schnitte." "Dieser Muskel hat also gar keine Ihnlichkeit mit einem Arrector. Er stellt anstatt dessen ein sehr unregelmäiig gestaltetes, in verschiedenen Richtungen geknicktes Bündel dar, das ganz frei in dem Bindegewebe liegt. In anderen Fällen ist jedoch die Ahnlichkeit des Muskels mit einem Arrector größer."**)

„Ob es aber etwa doch Muskelbündel gibt, die mit den Arrectoren nichts zu tun haben?"; diese Frage eruiert Sobotk $\left(t^{46}\right)$ in seinen Beiträgen zur Kenntnis der Cutismyome. ,In einer Peihe von Fälen vermag ich tatsächlich gewisse Bündel nicht nur nicht an einen Artector, sondern überhaupt an keine Muskelmassen zu verfolgen. Es handelt sich dabei ganz typischer Weise immer um zarte Züge, die nach ihrer Lage und Verlaufsrichtung an Arrectoren oder Arrectorenwurzeln erinnern, und die dann gewöhnlich in der Nähe größerer Muskelgebilde enden." "In meiner Darstellung des histologischen Befundes (der Cutismyome)", sagt So bo tka an anderer Stelle, , wagte ich nicht mit voller Sicherheit zu behaupten, daß Muskelzüge vorkämen, die in keinerlei Sinne eine Beziehrng zu den. Arrectoren

*) Im Auszuge und stark gekürzt. 
besalsen. Aber ein Bünlel, das an der Vorderseite des sonst arrectortonen Hibarbalges als feiner, dem Haarbalge paralleler strang verläuft, ohne daß weit und breit ein Haarmuskel anzutreffen wäre, von dem es abgespalten sein könnte, ein solches Bändel hat zum mindesten mit der normalen Hautmuskulatur nichts zu schaffen." "Schwer ist von einem Arrector von regelrechter Anlage auch folgendes, ganz groteskes Gebilde abzuteilen: Ein Muskelstrang von der normalen Breite eines Arrector zieht nicht mit normaler Schrägheit, sondern parallel zum Haarbalge und unmittelbar neben diesem und seiner kleinen, flachen Talgdrüse hin, sendet etwa in der Gleiche der Haarwuzel ein kräftiges Bündel in die Tiefo und biegt, ohne mit dem Haarbalge in nachweishare Beziehung getreten zu sein, um den Fundus der Talgdrüse und die Gegend der Haarwurzel in unverminderter Stärke alimïhlich wieder nach oben, strebt nun, auf der anderen, der Regel nach arrectorfreien Seite des Haarbalges, der Epidermis wieder $7 u$ und zwar in einer Richtung, die genau das Spiegelbild cler Richtung eines normalen Arrector ist, und zerlegt sich endlich, unter allmahlicher Verschmälenung, in eine ganze Anzahl von allerdings etwas unregelmäbig angeordneten ,Arrectorenwurzeln". Man kann sich des Gedankens kaum. erwehren, daß es sich bei diesen nicht recht erkläbaren Gebilden um Inomalien in dor Anlage der Arrectoren handelt."

Abweichungen rom normalen Verlaufe, Pichtungsinderungen, Zu- und $A b$ nahme der Länge und Dicke. Riesenarrectoren. Hypertrophic und Atrophie der Arrectoren wurden bei Durchsicht des mir zur Verfügung stehenden Materials, nicht selten gefunden (Abb. 5 und 6), wobei Bonnets ${ }^{5}$ ) und Rackows; $5^{36}$ Ergebnisse als zu Recht und als allgemein gültig betrachtet wurden, nämlich daB:

a) ,die Länge der schiefen Hautmuskeln einerseits im geraden Verhältnis zur Länge der zugehörigen Haarwurzel steht (Bonnet) und andererseits abhängig ist von der numerisch stärkeren oder schwächeren Behaarung, - je stärker der Zahl nach die Behaarung, desto steiler die Einpflanzung des Haarbalges, desto steiler und kürzer der Arrector pili und umgelkehrt" [Rackow*)],

b) "die Dicke der schiefen Hautmuskeln nicht im geraden Verhältnis zur Dicke des zugehörigen Haares steht, sondern zur Größenentwicklung der zugehörigen Drüsen, speziell der Knäueldrüsen" (Bonnet). Hierbei ist nach Rablan) zu bemerken, ,daB, wenn mehrere Haare zu einer Gruppe vereinigt, so ihre Muskeln häufig in ihrem mittleren Teile verschmolzen sind, so daB eine große Muskelmasse gebildet wird, die aber nach abwärts in ebenso viele Bündel auslüuft, als Haare beisammen stehen".

Während der normalen Anatomie des Mensehen Vorkommen und Verbreitung der glatten Muskulatur in der Haut nur an den erwähnten und genannten Stellen und in beschriebener Weise und Ausdehnung bekannt sind, lehrt die

*) Seuffert.1. behauptet sogar, daß in stellenweise dichter behaarten Hautpartien der Säugetiere die glatten Muskeln vollständig fehlen können, während bei regelmäßig und nicht zu dicht stehenden Haaren jeder einzelne Haarbalg in der Regel mit seinem eigenen Muskel versehen ist. Es scheint also nach Seuffert in den behaarten Hautpartien die Menge der glatten Muskeln und der Grad ihrer Verbreitung in Beziehung za der Dichtigkeit des Haarbesatzes zu stehen. In den unbehaarten Stellen der Haut dagegen waren mit einer einzigen, etwas zweifelhatten Ausnahme (Gresäßschwielen von Inuus) nirgends contractile Elemente zu seher. 
pathologische Anatomie ein weiteres und ausgedehnteres Vorkommen derselben, und zwar in Form der Drrmatoleiomyome.

Als Ausgangspunkt und Matrix der cutanen und subcutanen Leiomyome werden ron den Autoren, mit einer gewissen Wahrscheinlichkeit auf Grund der histologischen Befunde und auf Grund ron Vergleichungen angenommen:

a) Die natürlichen cutanen Muskellager und Muskelgeflechte der Haut, der Tunica dartos scroti (Myome dartique), der Namille, der großen Labien, des Penis;

b) Anomalien und Überentwicklung der $\mathrm{Mm}$. arrectores pilorum;

c) Die Tunica media der HautgefißBe (Arterien und Venen, auch die muskularen Elemente der Vasa vasorum);

d) Die Muskelbündel der Knüueldrüsen;

e) Gebilde, die in der Nathe der Haut liegen und glatte Wuskelfasern enthalten;

f) Abnorme Anlagebedingungen und lokale entwicklungsgeschichtliche Anomalien, durch embryonal und kongenital aberrierte und isolierte, normal nicht vorkommende, glatte Muskelkeime, die also sekundar durch Störung und Abweichung zur Zeit der Entwicklungsperiode in die Haut gelangen oder dort als "Cberbleibsel fötaler Bildungen" (Babes), besonders im Gebiet von embryonalen Spalten (Gesicht und Hals, fissurale Myome nach Babes) liegen bleiben.

Da (er unter f) angeführte Ausgangspunkt für die Entstohung der Dermatoleiomyome besonderes Interesse bietet, so mögen einige Autoren das Wort haben. Babes ${ }^{2}$ sagt hierzu: ,Es schließen sich solche Myome an, welche an Stellen zu finden sind, die de norma kein Muskelgerüst enthalten. Wenn wir aber in Betracht ziehen, daß gelegentlich an verschiedenen Stellen der Haut Muskelzüge gefunden wurden, so werden wir nicht anstehen, solche Bildungen hierher zu zählen" (nämlich zu den Myomen).

Nach Lieber'6) scheint ,ein Teil der entsprechenden Muskelmassen auch ohne zugehörige Haare in der Cutis gelegen zu sein. Ob es sich hier um eine Art versprengter Keime handelt, will er nicht entscheiden". An anderer Stelle sagt Lieber: „Unter denjenigen Autoren, welche ihre Myome von den Arrectoren ableiten, huldigt ein Teil der Anschauung, daß es sich hier nicht um gewöhnliche Arrectores als Ursprungsstelle der Myome handele, sondern daß versprengte solche als Matrix anzunehmen seien. Andere Autoren betonen, daB sie auch auBerhalb der Myome die Arrectores hypertrophisch fanden. Auch wir konnten dies verfolgen, und auch uns schienen manche dieser Arrectores entsprechenden hypertrophischen Yuskelzüge ohne clazugehörende Haare zu bestehen. Aber wir wollen dies nicht mit Bestimmtheit behaupten."

Nach Sobotk $a^{46}$ ) : „kann man kaum umhin, eine derartig gelagerte und angeordnete diffus: Muskelneubildung (Myom) auf eine Wucherung von ,freien Muskelfasern zurückzuführen, von der Art derjenigen, die auBer älteren Verfassern - kürzlich wieder $F i c k^{*}$ ) - gerade in der Cutis an der Streckseite des Oberschenkels nachgewiesen hat. Erinnert man sich nun aber, daß wiederum Babes in der Timgebung des Afters, des Nabels und des Ohres das inkonstante Vorkommen spärlicher Muskelzüge von der Lagerung derjenigen des tiefen Muskelgeflechts feststellen konnte, und daB Cnna in der Haut der Stim, der Wange und

*) Siehe Ehrmann und Fick, Einführung in das mikroskopische Studium der normalen und kranken Haut. Wien 1905, Holder. S. 68 . 
des Rückens nicht an bestimmte Gewebselemente gebundene Muskelfasern, etwa vorn Verlaufe des Arrector, gefunden hat, deren Vorhandensein allerdings von Koelliker nicht anerkannt wird, so wird man den Gedanken naheliegend finden, daß whl auch einmal ein umschriebenes Myom oder eine Gruppe von. solchen, wenigstens an gewissen Körperstellen, von dieser Art freier Muskelfasern den dusgang genommen haben könnte:"

Veranlassung zu eigenen, weiteren Untersuchungen gaben Präparate aus der Achselhaut von Hingerichteten (aus der Sammling des Herm Prof. Sobotta); Anregung bot die Arbeit von Häggqvist und die von ihm vertretene Anschauung des Zusammenhangs zwischen den Kältepunkten der Haut und den in der Haut liegenden, von ihm beschriebenen glatten Muskelbündeln. Als Material standen zur Verfügung zunächst die Präparate zu den Abb. 1-3, 5 und 6, der Sammlung des Herrn Prof. Sobotta entstammend, und Präparat zu Abb. 4, ron Herm Prof. Heiderich zur Verfügung gestellt*). Während Prïparat zu Abb. 1 der Wangenhaut entnommen, mit Carmin gefärbt (Dr. M. Heidenhain fec.), zeigen Abb. 2 und 3 Schnitte aus der Achselhöhle, Abb. 4-6 aus der Kopfhaut Dekapitierter; Präparat 2 ist in Zenkerscher, 3-6 in Müllerscher Flüssigkeit fixiert; alle, mit Ausnahme von Präparat 1, mit Hämatexylin-Eosin gefärbt.

Utber Material und Methodik eigener Untersuchungen ist zu bemerken: Das Material wurde der Bonner Anatomie überwiesenen Leichen (20) baldigst nach der Einlieferung entnommen. Die Stücke wurden an folgenden Stellen excidiert: Streckseite des Vorderarms und des Oberschenkels, Stirn (Mitte), Bauchwand (Linea alba), Achselhöhle. Es wurden diese Lokalitäten gewählt:

1. mit Rücksicht auf die von Neumann angegebene und oben angeführte Häufigkeitsskala der glatten Muskeln der Haut;

2. mit Rücksicht auf die von Krause, Landois ${ }^{24}$ ), Neumann, TignoloLutat $i^{53}$ ) festgestellte und ausgesprochene Tatsache, daß die glatten Muskelzüge an der Streckseite der Extremitäten stärker entwickelt sind als an der Beugeseite;

3. mit Rücksicht darauf, daB nach Neumann an jenen Lokalitäten, an denen das elastische Gewebe stärker entwickelt ist (Streckflache der Extremitäten), die Züge der glatten Muskelfasem ebentalls mächtiger entwickelt sind.

Die Hautstücke wurden in Zenker fixiert und nach Xylol-bzw. Heidenhain scher Schwefelkohlenstoffbehandlung in Paraffin gebettet. Die Färbung der 10 und $15, \mu$ dicken Schnitte erfolgte mit Hansens Hämatoxylin-Eosin und nach van-Gieson Methode. Die Sehnitte wurden stets senkrecht zur Oberflüche geführt und zwar sowohl parallel wie senkrecht zur Jokalen Spaltbarkeit der Haut.

Der Nachweis von glatter Muskulatur wurde zu erbringen versucht:

1. durch das tinktorielle Verhalten der fraglichen Gewebsbündel; (das Plasma nimmt die Färbung nach Hämatox.-Eosin gut an und färbt sich nach van Gieson gelb);

2. curch Nachweis der den glatten Muskelelementen eigenen morphologischen Merkmale (es sind ja namentlich Form und Anordnung der Kerne, die die Erkennung und Bestimmung der glatten Muskulatur ermöglichen).

*) Den Herren Prof. Sobotta und Heiderich sage ich an dieser Stelle für die guitige Überlassung der Präparate ergebensten Dank. 
Geachtet wurde stets:

1. auf das spezielle Verhalten der gefundenen glatten Muskelbündel,

2. auf ihre Verbreitung, Anordnung und auf ihr Verhalten zur Umgebung.

Ich gehe aus von den schon erwähnten, mir zur Verfügung gestellten Präparaten der Kopf-, Wangen- und Achselhaut des Menschen, welche in den Abb. $1-3,5$ and 6 zur Darstellung gebracht sind. Diese illustrieren das Yorkommen der bereits angefühten und beschriebenen Mr. tensores cutis obliqui (Lnna).

Die Abb. 1-3*) lassen in einem ganz ungewöhnlichen Maße, ein ausgedehntes, flächenhaft ausgebreitetes und netzförmig angeordnetes Muskellager erkennen, das diffus in der umgebenden Haut angeordnet ist und aus einer großen

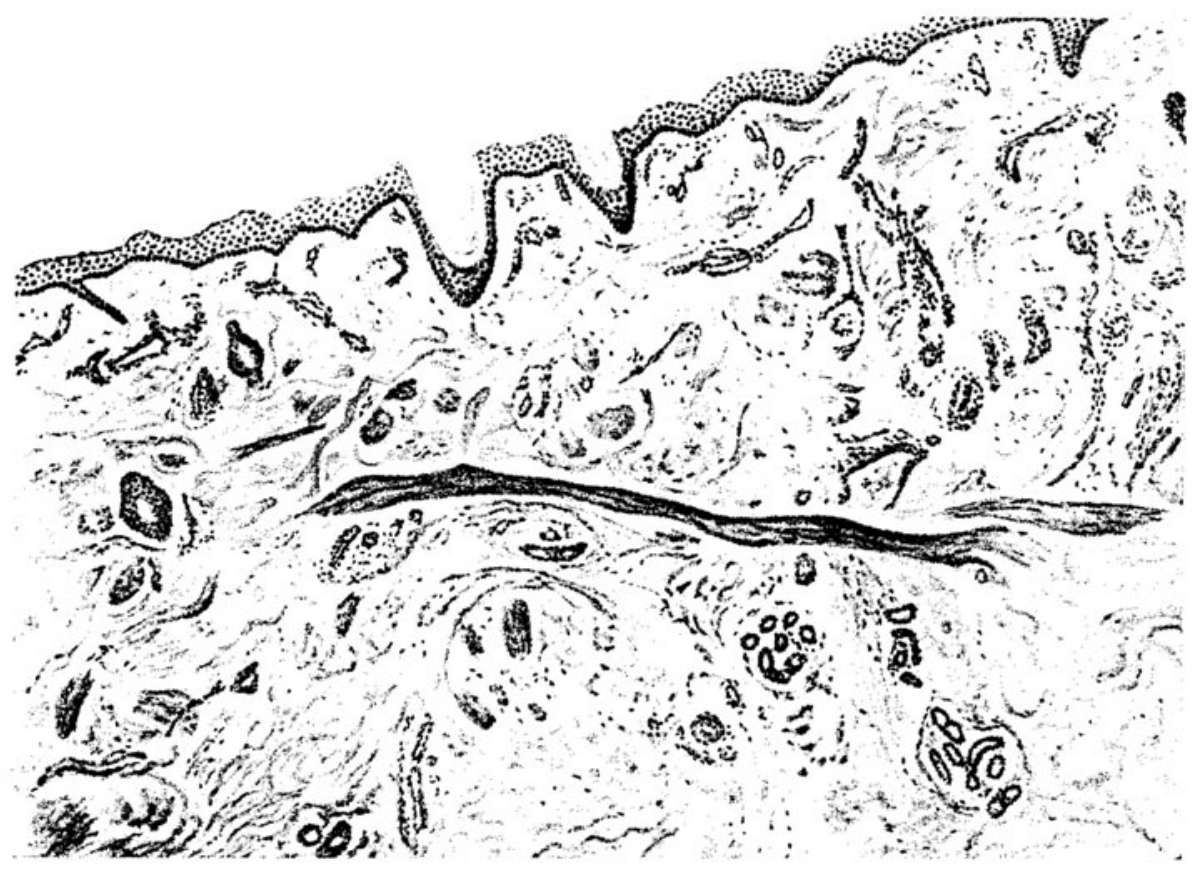

Abb. 1. Wangenhaut: Carmin (Dr. M. Heidenhain fec.), Zeiss, Okul. 4; Obj. a..

Zahl ron nach verschiedenen Richtungen verlaufenden glatten Muskelzügen besteht, die verschieden breit, in Längs- und Schrägschnitten getroffen sind, zwischen denen Querschnitte gelegen, welche Bündeln angehören, die zur Schnittrichtung senkrecht ziehen. Das Muskellager ist von einer solchen Mächtigkeit, wie man es sonst nur in den Membranae seu Tunicae musculares (Unna) zu finden pflegt and stellt eimen wesentlichen Bestandteil des Corium dar, vorwiegend dem Stratum reticulare, aber auch den tieferen Lagen des Stratum papillare angehörend, an einigen Stellen tief in die Cutis bis in die Nähe des Panniculus adiposus hineindringend. Zwischen den Muskelmassen liegen dichte, wellige Bindegewebsbündel, welche die Muskelzüge gewissermaBen in Form eines Netzes umspinnen. Die schmäleren und breiteren Bündel und Stränge, aus denen das Muskellager

*) Samtliche Abbildungen sind nach Mikrophotographien ausgeführt. 
zusammengesetzt ist, nehmen wahrend ihres Verlaufes eine wechselnde Richtung ein - diagonal durch den Dickendurchmesser der Haut bis fast parallel zur Oberflache (horizontal) verlaufend -, indem sie sich mehrfach in den verschiedensten Tbenen und Tiefen durchkreuzen, miteinander regellos verflechten, sich über- und durcheinanderschichten und schlingen und netzförmig untereinander anastomosieren, so daß eine Art von teils weit-, teils engmaschigem, dichtem Netz und Flechtwerk von Wuskelbalken, ein regelloses Wuskellabyrinth entsteht.

Füufig sicht man die Bündel an einzelnen Stellen anschwellen, um bald

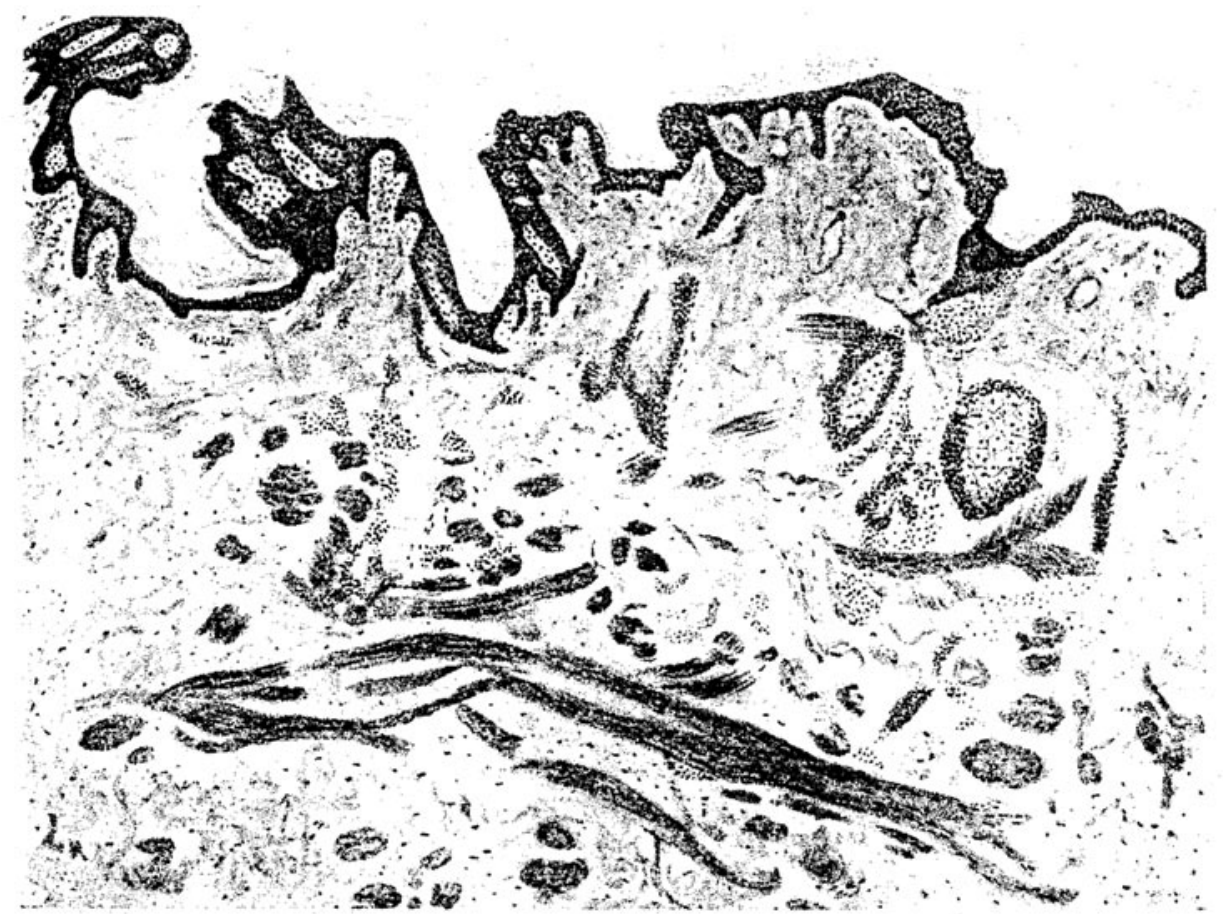

Abb. 2. Achselhöhle. Zeiss, Okul. 4, Obj. as. Frklärung der Abbifdungen im Text.

wieder abzunehmen, oder sich in einfache und geteilte Zweige und Aste spalten, wie von ihnen feine, zartere, gelegentlich auch mächtigere Sprossen and Ausläufer in wechselnder Höhe, oft zu mehreren nebeneinander liegend, abgehen, um sich an neve Bündel zu legen, die wiederum vielfach länglich ausgezogene Verzweigungen und Verästelungen entsenden, welche sowohl in horizontaler wie diagonaler Richtung verlaufen und zuweilen an einem Fnde, unter allmählicher Verschmälerung, pinsel- und fächerförmig ausstrahlen and in eine Zahl von Zipfeln sich zerlegen. In keinem Falle sieht man, daß die Fortsätze das Corpus papillare selbst erreichen. Andererseits sieht man nebeneinander liegende Züge sich vereinen und miteinander verschmelzen. Es stehen also die Bündel mit anderen, neben oder über bzw. unter ihnen liegenden, in höheren oder tieferen 
Schichten verlaufenden, gleichen Bildungen in Verbindung und in ramigfacher topographischer Beziehung zueinander.

Ein Zusammenhang der Nuskelmassen mit Mm. arrectores pilorum lronnte. in keinem Schnitto nachgewiesen werden; Harbälge fehlen in den Präparaten zu Abb. 2 und 3 völlig, während die Schweißdrüsen vorhanden und gut en twickelt sind.

In dem Präparat aus der Wangenhaut (Abb. 1) dagegen sind die Haarbälge, wakerbalb des Bereichs der Abbildung, vorhanden, aber keine typischen Mm.

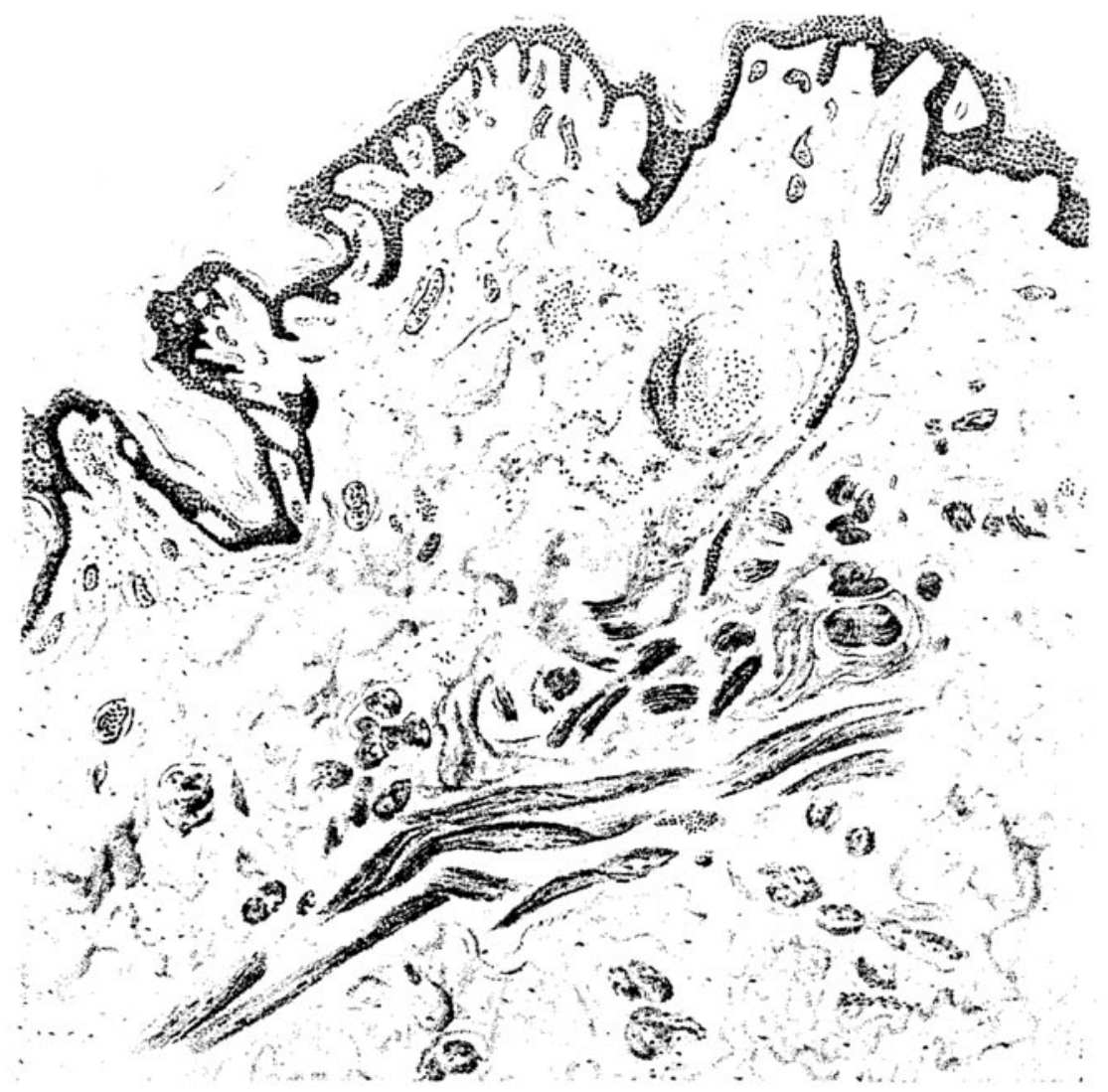

Abb. 3. Achsolhohle. Zeiss, Okul, 4, Ohj. as.

arrectores zu sehen. Eine Zunahme der Muskulatur der Gefiße und Drüsen konnte nicht nachgewiesen werden.

Es finden sich also in diesen Abbildungen, ebenso wie bei Newnann in der Achselhöhle, oberhalb and unterhalb der Schweißdrüsen, teils horizontal, teils in allen möglichen Richtungen verlaufende und schräg in die Cutis aufsteigende, anscheinend selbständige und ,freie" glatte Muskelbündel, die netzförmig angeordnet, ohne nachweisbaren Zusammenhang mit Haarbälgen und Arrectoren sind, Muskelbündel, die nach Neumann aber dennoch dem System der Arrectores pilorum angehören sollen. 
Es kann bereits hier festgestellt werden, claß die horizontal verlaufenden Muskelzüge und Stränge in der Achselhöhle nicht konstant vorkommen; schon Neumann sagt, der Befund scheine lokal variabel und individuell verschieden zu sein, was auch bei den eigenen Untersuchungen bestätigt wurde, denn in keinem der selbstverfertigten Präparate aus der Achselhöhle konnten Bildungen, ähnlich den beschriebenen, nachgewiesen werden.

Das Bild der in der Wangenhaut gefundenen Muskelelemente (Abb, 1) stimmt überein mit den Befunden bei Babes. Tomsa und Cinna, die ebenfalls Hautmuskeln des Gesichts, besonders der Backen- und Wangengegend fanden (Tomsas ,fragliche" Muskelnetze aus der Backengegend der Gesichtshaut des Menschen). Mit Mm, arrectores können diese Züge nichts zu tun haben, denn

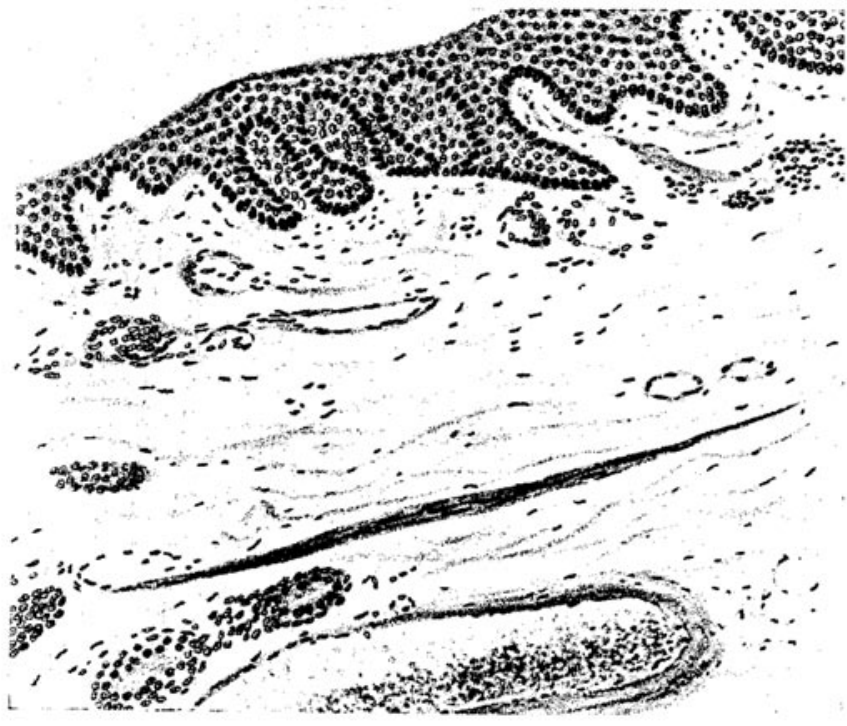

Abb. 4. Zeiss, Okul. 4, Obj. 8. nach Klemensiewicz haben die Haure der Wange überhaupt keine Arrectores, und in dem abgebildeten Präparate konnten auch einwandfreie Arrectores nicht nachgewiesen werden.

Daß es aber auch im Bindegewebe des Corium vereinzelt gelegene, zarte und schmale Züge von glatten Muskelfasern gibt - im Gegensatz zu den vorher beschriebenen, ausgedehnten Muskelmassen und den noch zu erwähnenden, breiten Zügen der Extremitäten -, die als feine Stränge, mit zur Hautoberfläche paralleler Zugrichtung, um $90^{\circ}$ von der Zugrichtung eines Arrector gedreht, stets in derselben Höhe und gleichen Lage des Stratum reticulare liegenbleiben, zeigt Abb. 4. Solche Streifen von glatter Muskulatur wurden nicht selten gefunden. Das Bündel gleicht in seiner Lage durchaus nicht einem Arrector, es ist auch nicht an einen Arrector zu verfolgen, besitzt in keinerlei Sinne Beziehung zu einem solchen; weit und breit ist ein M. arrector pili nicht anzutreffen, mit dern es zusammenhängen oder von dem es abgespalten sein könnte; es hat also mit einem. Arrector gar richts zu tun, erscheint vielmehr vollkommen selbständig.

So wie die bisher erwähnten and beschriebenen, zur Verfügung gestellten Präparate gaben auch die eigenen, selbstwerfertigten durchaus eine Bestatigung und Erweiterung der in der Literatur niedergelegten Ergebnisse. In zwei Hantstücken, der Mitte der Stirn entnommen, wurden in den obersten Schichten und Teilen des Stratum reticulare und dem Grenzgebiet zwischen diesem und dem 
Stratum papillare gelegene, schrïg und horizontal verlaufende glatte Muskelzüge gefunden, die keinen Haaren zugehörten und in keiner direkten Beziehung zu Mm. arrectores standen. Schweißglusen waren reichlich und gut ausgebildet vorhanden. Es liegen also auch hier, entsprechend dem mikroskopischen Bilde, ,freie" Muskelzüge vor, denn auch in der Stirn fehlen nach Klemensiewicz die Mm. arrectores, wie ja in allen Gegenden, die reich sind an anderen glatten und quergestreiften Muskelfasern. Dreimal wurden in der Haut des Vorderarms und zweimal in der Haut des Oberschenkels bei verschiedenen Individuen einzelne dünne oder krüftigere glatte Muskelzüge gefunden, die auf den ersten Blick von den $\mathrm{Mm}$. arrectores pilorum durch ihre Verlaufsrichtung sich unterschieden. Diese unregelmäßig gestalteten und in verschiedenen Richtungen geknickten Züge zeigen nämlich nicht die normale, perpendikuläre und diagonale Lagerung durch die Dicke der Haut, wie sie den Mm. arrectores in ihrem ganzen Verlaufe eigen, sondern sind mehr oder weniger horizontal, stellenweise oberflächenparallel. Die Bündel nehmen das Corium in verschiedenen tusmaße und in verschiedener Tiefe ein, sind eingelagert in die oberen zwei Drittel des Stratum reti. culare und in die Grenzschicht zwischen Stratum reticulare und Stratum papillare, sind teils dichter, teils weniger dicht und dann durch Bindegewebszüge voneinander getrennt. Die Breite dieser atypisch verlaufenden Bündel ist stellenweise ungetânr die gleiche wie die eines Arrector, stellenweise sehr beträchtlich, stellenweise aber auch gering. Nirgendwo ist ein Zusammenhang der Züge mit den $\mathrm{Mm}$. arrectores pilorum nachweisbar : es handelt sich demnach mit der größten Wahrscheinlichkeit um Bündel und Stränge von selbständigem Charakter, frei im Bindegewebe gelegen, weder mit dem Corpus papillare noch mit einem Haar. follikel in Verbindung tretend. Schweißdrüsen und Haarbälge sind dabei vorhanden.

Diese Befunde entsprechen denen Neumanns, der ja ebenfalls an den Streckflächen der Extremitaten, in dem oberen Teile des Corium, horizontale, breite Züge von Muskelfasern gefunden hat, die selbständig sind und mit Muskeln des Haarbalges nichts gemein haben. An dieser Stelle sei nochmals darauf hingewiesen, dal, im Gegensatz zu Neumann, Krause an der Vorderflaiche des Oberschenkels sich horizontal, an den Schweißdrüsen vorbei erstreckende Ausläufer der Arrectores gesehen hat (s. o.).

Alle diese bisher beschriebenen Muskelzüge sind als $\mathrm{MLn}$. tensores cutis obliqui, als schrïge Hautspanner (Unna), anzusprechen. Und da es nicht erwiesen erscheint, daß diese muskulären Bildungen von den Arrectores herrühren, zumal ja auch die Verlaufsrichtung - mehr oder weniger oberflächenparallel - dagegen zu sprechen scheint, und ein fixer Anhaltspunkt weder an einen Haarfollikel noch an das Corpus papillare nicht nachweisbar ist, so sind die Muskelzüge demnach in den meisten Fällen wohl als $\mathrm{Mm}$. cutis diagonales (Unna) zu rubrizieren. Wenn nun auch die Zugehörigkeit der gefundenen Muskelmassen und -züge zu dem System der Mm. arrectores pilorum mit Bestimmtheit nirgendwo nachgewiesen werden konnte, so mu $B$ doch andererseits zugegeben werden, da $B$ die Ableitbarkeit von diesem wenigstens möglich ist. Schon Krause sah ja manche Auslaufer der Arrectores tiefer in die Cutis reichen und sich mehr horizontal an den SchweiBdrüsen vorbei erstrecken, und anch in der Achselhöhle und in der be- 
haarten Kopfhaut kommen nach ihm mitunter solche Bündel vor, und Neumann ist es, , in vielen Fallen zweifelhaft, ob es sich hierbei um selbständige Muskelfasern handelt oder vielleicht um Zweige der Arrectores pilorum, um so mehr. als diese sich vielfach verzweigen können".

Die $\mathrm{Abb} .5$ und 6 stellen glatte Muskelzüge dar, wahrscheinlich Arrectores pilorum, von atypischer Verlaufsrichtung.

Abb. $\check{0}$ zeigt ein im Schrägschnitt getroffenes, leicht $\$$-fömig gebogenes

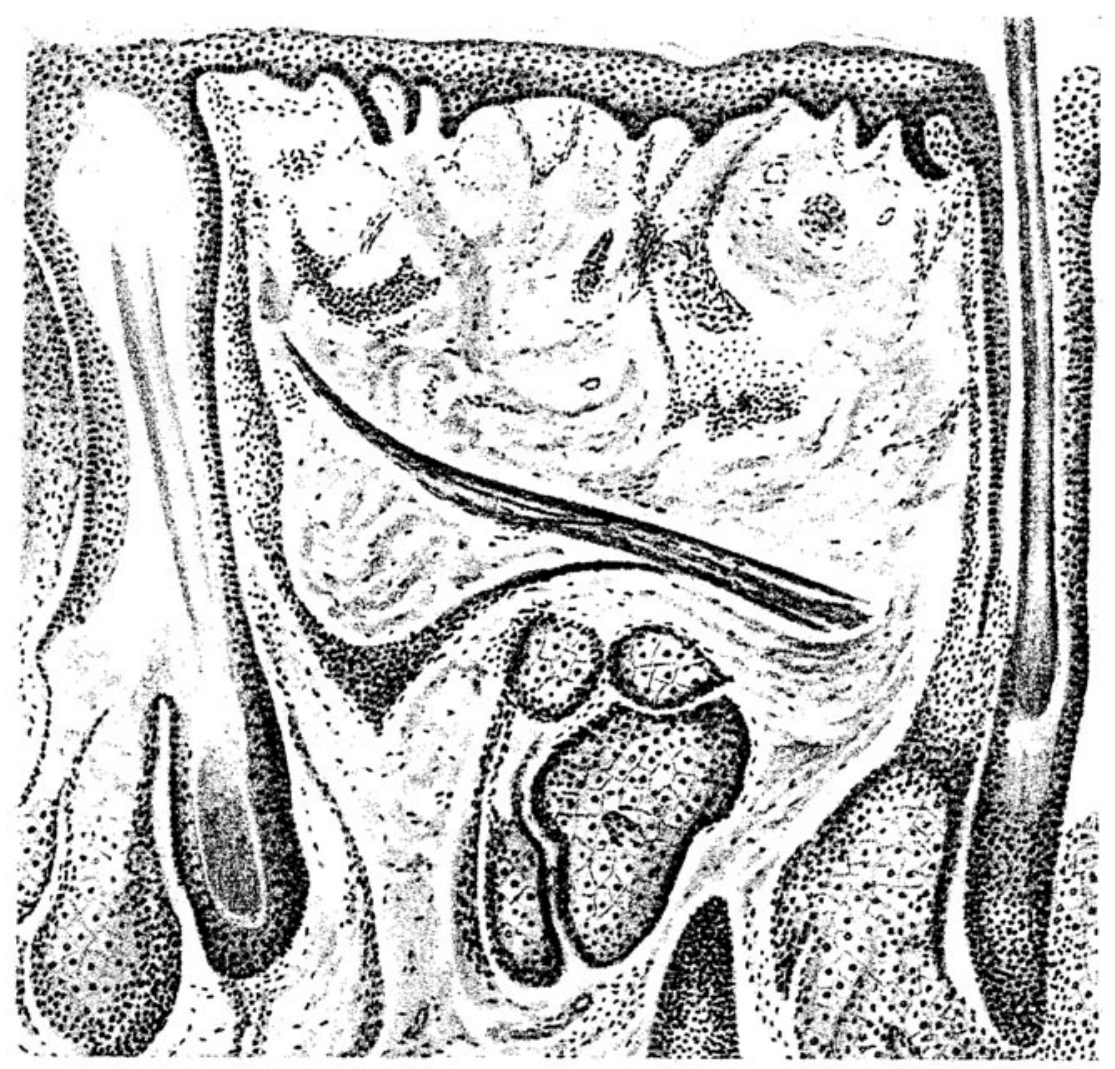

Abb. 5. Kophaut. Zeiss, Komp.-Okul. 2, Obj. 16.

glattes Muskelbündel von der normalen Breite eines Arrector, das gegen die Hautoberfläche eine sehr geneigte und schräge Richtung einnimmt, in dieser Richtung dem Stratum papillare zustrebt und das, wie mit gröBter Wahrscheinlichkeit anzunehmen ist, infolge seiner Lagerung zwischen Haarbälgen und oberhalb von Talgdrüsen, wohl als mit diesen in Verbindung stehend betrachtet werden muf, wenn auch eine Verbindung und nachweisbare Beziehung zu dem Haarbalge und dem Corpus papillare in der Abb. nicht zu sehen sind. Auch ist die Ahnlichkeit des Bündels mit einem Arrector groß und auf den ersten Blick ins Auge fallend. Die Abbildung demonstriert also einen nahezu horizontal und oberflächenparallel verlaufenden glatten Muskelzug in der Haut, 
der mit großex Wahrscheinlichkcit mit einem Arrector in Verbindung und Zusammenhang steht oder gar selbst einen Arrector darstellt, der von seinem normalen, perpendikulären Verlaufe abgewichen und seine Pichtung geändert hat.

Der Arrector der Abh. 6, der zwischen zwei Haarbälgen gelegen und dessen oberhab der stark entwickelten Haarbalgdrüsen, in den mittleren und oberen Abschnitten des Stratum reticulare befindlicher Abschnitt wiedergegeben ist, zeigt eine, die normale Breite übertreffende Mächtigkeit. Aber nicht nur diese alle normal vorkommenden Haarbalgdrüsenmuskeln übertreffende, ungewöhnliche Stärke und mächtige Entwicklung des Arrector sind von Interesse; sondern auch seine starkwinklige Umbiegung und gabelförmige Verzweigung, seine fächerförmige Verästelung und Ausbreitung in zwei Zipfel (nach links im Bilde) sind besonders hervorzuheben, ebenso wie die Tatbache, daß der Muskel an der Lmbiegungs-, Teilungs- und Spaltungsstelle, an der Grenze zwisehen oberer and mittlerer Sohicht des Stratam reticulare (von rechts in Bilde aus), einen horizontal verlaufenden, krïftigen Muskelzweig und Nebenast aufnimmt, dessen Ursprung und Herkunft nicht klar sind. Demnach lehrt diese Abbildang, daB es Falle gibt, in denen horizontal verlaufende, oberflächenparallele glatte Wuskelbündel nachweisbar mit einem Arrector in Verbindung treten.

Die Frage, ob die in das Derma eingelagerten, untersuchten und beschriebenen Muskelmassen und Bündel

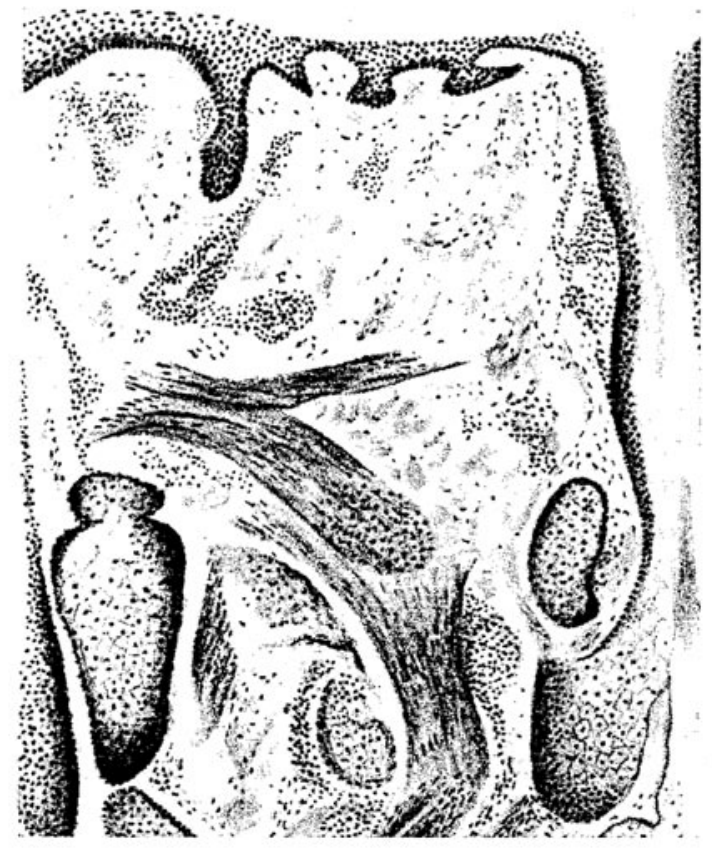

Abb. 6. Kopthat. Winkel, Okul. 1, Obj. 2. Abkömmlinge der präexistierenden muskulären Elemente der Haut, der Mm. arrectores pilorum, oder ,freier" ${ }^{\text {" }}$, selbständiger Natur sind, scheint mir demnach weder nach der einen noch nach der anderen Seite, weder in bejahendem noch in verneinendem Sinne, mit Bestimmtheit beantwortet werden za können, solange nicht ein unmittelbarer Zusammenhang zwischen beiden, abnlich wie die in $\mathrm{Abb} .6$ besehriebene, direkte Verbindung zwischen den Muskekzügen, im mikroskopischen Bilde einwandfrei sichergestellt werden kann. Wenn auch die Möglichkeit des Zusammenhangs bzw. der Ableitbarkeit von Nm. arrectores pilorum in einigen Fällen zugestanden werden muB (s. oben Krause, Neumann), wenn wach einge Grüde für die Annahme dieses Zusammenhangs oder einer Abstammung von den $\mathrm{Mm}$. arrectores pilorum zu sprechen scheinen, so das überwiegende Vorkommen der geschilderten Befunde an Stellen, an denen 
die Mm. arrectores pilorum am häufigsten und stärksten entwickelt (Streckfläche der Extremitäten), und das Fehlen derselben an Stellen, an denen Mm. arrectores pilorum nicht vorhanden (Vola manus, Planta pedis, Hüygqvist). so findet der Befund in der Achselhöhle dadurch jedoch keine Erklitrung, auch die andere Verlaufsrichtung der Muskelzüge spricht dagegen, und die bisherigen anatomischen Untersuchnngen sowie die Ergebnisse der Literatur lassen vermuten, daß eine solche Verbindung im allgemeinen nicht zu bestehen braucht. Das nicht konstante und relativ seltene Vorkommen des, lokal variablen und individuell verschiedenen" Befundes, die unbeschränkte Wachstumsmöglichkeit an beschränkter Stelle (Achselhöhle) sind Tatsachen, die nahelegen, raß es sich möglicherweise um eine lokale Entwicklungsanomalie handelt, entsprechend der von einer Reihe von Autoren für die Entstehung der Dermatoleiomyome verantwortich gemachten. Das Fehlen solcher Muskelmassen und Büdlel beim Neugeborenen und Jugendlichen (Neumann) spricht nicht dagegen*), denn die Wucherungsfähigkeit der versprengten und isolierten Keime liann etwas "Latentes darstellen, das erst später labil und ausgelöst wird" (Babes). Oder sollte es sich um eine rudimentäre Anlage handeln, um eine :: Erinnerung: an das ausgedehnte Vorkommen und die Verbreitung von glatter Muskulatur in der behaarten Haut vieler Säuger? [Seuffert44)].

Nach Häggqvists Theorie hat der von ihm beschriebene Nuskel nicht nur die Aufgabe, ,das Haar aufzurichten und das Selret der Talgdrüsen auszupressen, sondern er muß auch eine andere dufgabe haben oder eine neue bekommen, wenn er die beiden erwähnten nicht mehr haben kann". H. kommt zu diesem Schlusse, die Tatsache berücksichtigend, ,daß mm. arrectores pilorum nicht atrophieren, wenn die Haarbälge zugrunde gehen". Diese neue Funktion des Muskels besteht nach $H$. nun darin, da $B$ er, wenn durch direkte $K$ ältereizung oder wahrscheinlicher auf dem Reflexwege zur Kontraktion gebracht, ,die regionäre Blutzufuhr zur Haut verhindert, was eine geringere Verbrennung in den äußeren Lagen der Haut verursachen muB::H. nimmt nämlich einen Zusammenhang $z$ wischen den von ihm beschriebenen glatten Muskelbündeln der Haut und den Kältepunkten an und begründet seine Anschauung damit, da $B$ er cliesen Muskel nur in Hautstücken gefunden habe, die einen Kältepunkt enthielten, dagegen nie in Hautstücken ohne solehe.

Das Bestehen eines solchen Zusammenhanges erscheint mir aber nicht sehr wahrscheinlich. $H$. sagt selbst, er habe ein solches Bündel glatter Muskulatur nie gefunden in der Vola manus und Planta pedis - Lokalitäten, in denen doch Kältepunkte vorhanden sind. Ein weiterer Grund, der eine solche Relation fraglich erscheinen läßt, scheint mir in dem Mißverhältnis gelegen zu sein zwischen dem Vorkommen der Kältepunkte einerseits - ihre Anzahl beträgt ungefähr 10 pro qcm, sie liegen im Mittel $3 \mathrm{~mm}$ voneinander entfernt (Häggqvist) -

*) Liach AbschluB dieser Arbeit wurde mir ein in hiesigen histologrischen Laboratorium Fon Herm Dr. med. Beclerr vertertigtes Präparat eines 3 Monate alten männlichen Kindes rorgelegt, der Haut in der Witte der rechten Bicepsgegend entnommen. das deutlich breite und krättige, hypertrophische glatte Muskelzüge orkennen ließ. die von atypischer, teils obertläehenparalleler Verlaufsrichtung, an einigen Stellen gespalten und verästelt, einen nicht selbständisen Charakter trugen. 
und dem Torkommen dex ,freien" glatten Muskulatur der Hant anderetseits, die durchaus nicht, nach meinen Untersuchungen, eine solche Verbreitung aufweist, wie $H$. es mitteilt. sondern inkonstante, variable und individuell ferschiedene Befunde zeigt.

\section{Zusammenfassung.}

I. Die in den Lehr- and Handbüchern verbreitete Ansicht über das Vorkommen der glatten Muskulatur in der Haut cles Menschen ist eine zu enge. Denn es finden sich dort - die Fleischhäute ausgenommen - zumeist nur Beschreibungen derjenigen glatten Mruskeln der Haut, welche mit Haaren in Ferbindung stehen.

II. Ton einer Reihe von Autoren sind in der Haut des Menschen glatte Muskelelemente gefunden worden, die nicht an Haarbälge gebunden sind. Das Vorkommen solcher, freier und selbständiger" glatten Muskelelemente konnte in einer Reihe von Fallen nachgewiesen und bestatigt werden, so dab demach mit Veumann zu Pecht behauptet werden kann: .Die glatten Muskein der Huwt sind in größerem Maßstabe verbreitet, als man bisher geglaubt hat."

III. Gestützt auf Unna und eigene Cntersuchungen lassen kich folgende Gruppen ron glatten Hautmuskeln (nach $(n n a$ ) aufstellen:

a) Membranae seu Tunicae musculares (Torkommen: Tunica dartos scroti, Penis, Perineum, Mamilla und nach Babes unbedeutende Zïge im Gesicht, in der Umgebung des Afters, Nabels und Ohres).

b) $\mathrm{Mm}$. tensores cutis obliqui:

1. Mm. erectores pilorum (Vorkommen: an allen behaarten Hantstellen; Ausnahmen nach Diesing und Klemensiewica);

2. Mm. cutis diagonales [Vorkommen: siehe Babes, Dariers), Häggrvist,

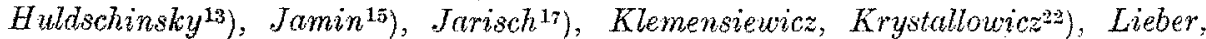
Neumann, Pasini33), Sobotka, Tomsa, Linna, Fignolo-Lutati; beschriebene Befunde in der Achselhöhle, Wange, Kopthaut, Stim, Streckflache der Extremitäten].

IV. Das Vorkommen der $\mathrm{Mm}$. cutis diagonales ist nicht konstant, sondern sehr variabel und individuell versehieden. Es scheinen gewisse Prädilektionsstellen (Streckseite der Extremitaten, jene Lokalitäten, an denen das elastische Gewebe stärker entwickelt ist) zu bestehen (Krause, Landois, Neumann, Quast, Vignolo-Lutati).

Tch erfülle eine angenehme Pflicht, indem ich Herrn Prof. Dr. Sobotta für die Uberweisung der vorliegenden Arbeit und für die freundliche Unterstütung bei ihrer Anfertigung ergebensten Dank sage.

\section{Literaturverzeichnis.}

1) Babes. F., Ein subeutanes Myom der Hohlhand. Ziemssens Handbuch der speziellen Pathologrie und Therapie 14. 1884. - I) Babsa, $F_{.,}$. Die dem Arehiblast der Haut angehörigen Geschwalste. Das Mrom. Ans Schwimmer and Babes: Die Neubildungen der Haut in $\mathbb{H}$. $\nabla$.

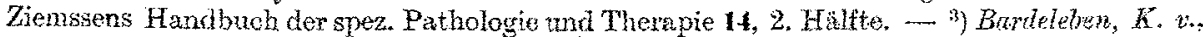
Lehrbuch der systematischen Anatomie des Mensehen. Berlin 1906. - - i) Böh and Davidoff, Lehrbuch der Histologie des Menschen. 2. Aufl. 1898. Verlag Bergmann. - 5) Bonnet, Uber 
die glatte Muskulatur der Haut und dex Knäueldrisen. Arrti. Intellitenzhlatt Nr. 3. 185.5. - b) Borst. M., Die Lehre von den Geschwïsten. Wiesbaden 1902. Bergmann. -- i) Brisikike. Gustan, Lehrbuch der normalen Anatomic des menschlichen Körpers. 9. Aufl. Berlin 1912. - 8) Drrier. Tumeur de la peati. La pratique dernat. Bd. 4, 1304. - Diexing, $R$., Beiträge zur Kenntnis ler Haarbalgmuskein. Beitrage zur Morphologie und Worphogenie, hrsgeb. von Gerlach 1883. Bd. 1 [zit, nach Rablas]]. - ig) Gegenbuar. Fürbringer. Lehrbuch cler Anatomie der Menschen. Leipgig 1909. 8. Aufl. Bd. 1. - ${ }^{11}$ ) Gösta-Häggr rist. Histophysiologische Studien uber die Temperatursinne der Haut des Menschen. Aus der histologischen Abteilung des Carolinischen medico-chirurgischen Instituts in stockholm. Anat. Inz. 45. Xr. 2 u. 3. 1914. 12) Hesse. Fr. Zur Kenntnis der Hautdrüsen und inrer Muskeln. Zeitsehr. f. Anat. a. Entwicklungsgesch. :. 1876. - 13) Hulll.schin.sk\%. K. Ein Beitrag zur Kenntnis der multiplen Dermatomyome. Freiburger Diss. 1901. - 14) Jatassohn. J., Zur Kenntnis der multiplen Myome der Haut. Virchows Arch. f. pathol. Anat. u. Physiol 121. 1390. - 13j Jamim, F., Ein Fall von multiplen Dermatomyomen. Dtsch. Arch. f. klin. Med. 70. 1901. - $\left.{ }^{16}\right)$ Jarisch. A.. Ferhandlung der Deutschen dermat. Gesellschaft, $\hat{0}$. KongreB, 1895. - 17) J(crisch. A.. Die Hantkrankheiten. In Spezielle Pathologie und Therapie, hrsgeb. von H. Nothnagel. Wien 1900. Bd. 24. Abt. 1. - ${ }^{18}$ ) Kaufmann, E., Lehrbuch der speziellen pathologischen Anatomie. Berlin 1900. Georg Reimer. - ${ }^{19}$ ) Klemensiewicz. Haut und Hautorgane in A. Eulenbury, Realenzyklopädie der gesamten Heillinde. 3. Aufl. 10. Bd. 1896. - - ${ }^{20}$ ) Koelliker, A., und V. v. Ebner, Handbuch der Gewebslehre des Menschen. Leipzig 1889-1902. 6. Aufl. Bd. I. 21) Koelliker, A.. Beiträge zur Kenntnis der glatten Mriskeln. Zeitschr. f. Wiss. Zool., hrsgegeb. ron Siebold und Koelliker. 1848. 1. Bd. - 22) Krystallowice. Multiple Hautmyone. Przeglad lekarsky Ar. 49, 1905; zit. nach .J. Steinhwhs. Polnische Literatur in Lubarsch und Ostertags Ergebnisse der allgemeinen Pathologie und patholog. Anatomie 11. 1907. 2. Teil. - ${ }^{23}$ ) Krystallowicz, Ein Fall von Myomatosis cutis disseminata. Monatsh. f. prakt. Dermat. 12. 1906. - 24) Lantois, L.. Lehrbuch der Physiolorie des Venschen. Bearbeitet ron R. Rosemann. Bd. 1. 13. Aufl. 1013. - san Langer; C. 2 , Lehrbuch der system. und topogr. Anatomie. Hrsgegeb. von C. Toldt. 10. Aufl. 1915. Wien und Leipzig. - ${ }^{25}$ Lieber, Farl, ther die Myome der Haut. In Beiträge zur pathologisehen Anatomiv und zur all-

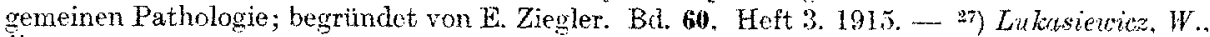
Uber multiple Dermatomyome. Arch. f. Derm. u. Syph. 24. 1892. - as) Merkel. Fr., Die Anatomie des Menschen. 5. Abtl. 1917. Texthand. Wiesbaden, Bergmann. _- 29) Yeumann. J.. ťber multiple Bermatomyome. Arch. f. Derm. u. Syph. 39. 1897. - ${ }^{30}$ ) Nemann, J., Über die Verbreitnng der organischen Muskelfasern in der Haut des Menschen. Allgem. Wiener med. Zeit. Xr. 29 u. 30. 1868. - 31) Veumunn. J.. Sitzungsberichte der Kais. Akademie der Wissensehaften zu Wien. Mathemat.-naturwiss. Klasse 5y, Abtl. 2. 1868. - * * Neumann, $f$, Osterreich. Zeitschr. f. prakt. Heilkunde. Heransgegeb. vom Doktorenkollegium d. Wiener mediz. Fakultat. 1t. Jahrgang, 1868. Nr. 19 a. 34. - 32a) Neumunn.J., Siehe auch Jahresberichte der gesamten Medizin. Herausgegeb. von Virchow-Hirseh. 1888. 1, Bd. 33) Ptasini, A., Uther einen Fall von angiokavernösem Myon der Haut. Monatsh. f. prakt. Dermat. 4, Haft 12. 1907. - $\left.{ }^{34}\right)$ Rabl, H., Haut. Ergebnisse der Aratomie und Entwicklungsgeschichte 7, Abt. 2. 1897. - 35$)$ Rabl. H., Histologie der normalen Haut in Franz Mraceks Handbuch der Hautkrankheiten, 1. Bd. 1902. Wien, Hölder. - 36) Rackow, J., Beitrag zur Histologie und Physiologie des glatten Hautmuskels des Pferdes. Arch. f. wissensehaftl. und prakt. Tierheilk. 24, Heft 3 und 4, 1898. — i7) Ramber-Kopsch, Lehrbuch der Anatomie des Menschen. 9. Aufl. 1912. Abtl. 6.- 38) Ribbert. H., Lehrbuch der allgemeinen Pathologie und pathologischen Anatomie. 5. Aufl. 1915. Leipzig, Vogel. - $\left.{ }^{39}\right)$ Ribbert, H., Geschwulstlehre. Bonn 1914. Friedrich Cohen. - ${ }^{40)}$ Rieh, $G$., Allgemeine Dermatologie in E. Rieckes Lehrbuch der Haut- und Geschlechtskrankheiten. 3. Aufl. 1914. 1. Teil. - $\left.{ }^{41}\right)$ Roux, $C$., Beiträge zur Kenntnis der Aftermuskulatur des Menschen. Arch. f. mikroskopische Anatomie 19, 1881 ; zit. nach $\left.R a b b^{* 5}\right)$. - 49) Schmaus-Herzheimer, H., GrundriB der pathologischen Anatomie. 10. Aufl. Wiesbaden 1912. - S3ymonowicz, L., Lehrbuch der Histologie und der mikroskopischen Anatomie. 2. Aufl. Würzburg 1909. - 4a) Senffert. L.. Cber das Vorkommen und Verhalten glatter Musheln in der Haut der Saugetiere und Vögel. Würzburger naturwissenschaftliche Zeitschr. 3, Heft 2. 1862. - ${ }^{45}$ ) Sobotka, P., Zur Kenntnis der Myome der Cutis und der Subeutis. Arch. f. Derm. n. Syph. 115 und 116. 1913; siehe auch Monatsh. 
f. prakt. Dermatol. 36. 1913. - *ij Sibothw. P.. Klin., histolog. u. vercleichende Beiträge zur Fenntnis der Cutismyone. Arch. f. Dermatol. u. Syphilis 89. 1908. - ${ }^{47}$ ) Sobotte, f., Atlas der deskriptiven tnatomie des Menschen. Bd. 3. 2. Aufl. Lehmann, Mïnchen 1916. 4) Sobotta. f., Atlas der Histologie. Lelmann. Minchen. - ${ }^{49}$ ) Stohr. Ph. Lehrbuch der Histologie, bearbeitet von O. Schuitze. 16. Aufl. 1915. - ${ }^{59}$ ) Siricker, S. Handb. der Lehre ron den Geweben des Menschen und der Tiere. Leipzir 1871-72. - 51) Tomsa. W. Beiträge zux Anatomie und Physiologie der menschlichen Haut. Arch. f. Demnatol. a. Syphilis .7. 1873. - it a) Toms $a$. $W$., Siehe auch Jahresberichte der gesamten Nedizin, herausgegeb. von Virchow-Hirsch. 1873. Bd. 1. - Un Una, Entwicklungsgeschichte und Anatomie der Haut. Aus Ennas Handbuch der Fautirankheiten. In Handbueh der speziellen Pathologie und Therapie. Herausgegeb. von H. v. Ziemssen. Leipzig 1883. Bd. 14. 1. und 2. Hälfte.5) Cuslo Vignoli-Lutati. Lxperimentelle Beiträge zur Pathologie der glatten Muskulatur der Hant, Areh. f. Dermatol. u. Syphilis 5\%. 1901.

\section{Nachtrag.}

Nach Abschlub der Arbeit wurde ich durch Herrn Prof. Sobolia auf eine im Biologischen Zentralblatt 1917, 37 erschienene Publikation von $P$. Schielferdecker aufmerksam gemacht über "Die Hautdrüsen des Menschen und der Säugetiere, ihre biologische und rassenanatomische Bedeutung, sowie die Muscularis sexualis", die einige das Thema vorliegender Arbeit berührende Mitteilungen enthält, welche sich ergänzend meinen Ergebnissen und Befunden (s. o. mm. cutis diagonales) einreihen. Sch. erwähnt, daB, an bestimmten Hautstellen des Menschen auch sonst im allgemeinen ziemlich oberflächenparallele Züge glatter Muskelfasern, gewöhnlich nur in einer bestimmten Richtung verlaufend, unabhängig von den Haaren und Drüsen, im Corium und (oder) in der Subcutis, selbst in mehreren Schichten übereinander auftreten können; auch Verbindungen der Schichten und Bündel kommen vor, die mitunter so dicht sind, dab sie an Durchflechtungen erinnern".

Uber Auftreten und Vorkommen dieser glatten Hautmuskulatur gibt Sch, an:

1. Mitunter die Achselhöhle (im Corium);

2. die "regio sexualis" (Schiefferdecker), in dieser

a) als "muscularis mamillae et areolae"s $(S c h)$,

b) als "muscularis sexualis" $(8 \mathrm{ch})$, vom scrotum, resp. den labia majora, verschieden weit und in verschiedener Stärke ausstrahlend auf den Damm, penis (namentlich dessen untere Seite), mons pubis. 